\title{
Quantum Monte Carlo calculations of van der Waals interactions between aromatic benzene rings
}

\author{
Sam Azadi* \\ Department of Physics, Imperial College London, SW7 2AZ London, United Kingdom \\ T. D. Kühne \\ Department of Chemistry and Paderborn Center for Parallel Computing, University of Paderborn, Warburger Strasse 100, \\ D-33098 Paderborn, Germany \\ and Center for Sustainable Systems Design and Institute for Lightweight Design with Hybrid Systems, Warburger Strasse 100, \\ D-33098 Paderborn, Germany
}

(Received 19 December 2017; revised manuscript received 27 March 2018; published 17 May 2018)

\begin{abstract}
The magnitude of finite-size effects and Coulomb interactions in quantum Monte Carlo simulations of van der Waals interactions between weakly bonded benzene molecules are investigated. To that extent, two trial wave functions of the Slater-Jastrow and Backflow-Slater-Jastrow types are employed to calculate the energy-volume equation of state. We assess the impact of the backflow coordinate transformation on the nonlocal correlation energy. We found that the effect of finite-size errors in quantum Monte Carlo calculations on energy differences is particularly large and may even be more important than the employed trial wave function. In addition to the cohesive energy, the singlet excitonic energy gap and the energy gap renormalization of crystalline benzene at different densities are computed.
\end{abstract}

DOI: 10.1103/PhysRevB.97.205428

\section{INTRODUCTION}

In his original work of 1930 [1], London introduced the fragment-based approach to van der Waals (vdW) interactions. As a consequence, the dispersion energy between two spherical objects $A$ and $B$ can be calculated using second-order perturbation theory for the Coulomb interaction. In its general form, the vdW energy $E_{v d W}$ can be written as

$$
E_{v d W}=-\sum_{n=6,8,10, \ldots} f_{n}\left(\mathbf{R}_{A B}, \mathbf{r}_{c, A B}\right) \frac{C_{n}}{\mathbf{R}_{A B}^{n}},
$$

where $f_{n}\left(\mathbf{R}_{A B}, \mathbf{r}_{c, A B}\right)$ is a damping function, which depends on a cutoff distance $\mathbf{r}_{C, A B}$, whereas $\mathbf{R}_{A B}$ is the distance between two fragments. The damping function attenuates the $\mathrm{vdW}$ energy for small values of $\mathbf{R}_{A B}$, where the electron clouds around fragments overlap [2,3]. The dipolar term $C_{6} / \mathbf{R}_{A B}^{n}$ of the London expression has been widely used for computing the vdW interactions within approximate first-principles electronic structure techniques, including Hartree-Fock (HF) [4,5] and density functional theory (DFT)-based methods [6-11]. Although, this description of vdW energy is particularly accurate in the far-field for which the overlap between orbitals of the fragments is small, it is nevertheless very practical to improve the accuracy of generalized gradient approximation (GGA) exchange and correlation (XC) functionals. In fact, it has been demonstrated that the inclusion of long-range dispersion effects systematically improves the description of nonlocal intermolecular interactions [9].

The so-called van der Waals density functional (vdW-DF) approach has been extensively used to correct conventional

\footnotetext{
*s.azadi@imperial.ac.uk
}

local and semilocal GGA XC functionals [12-14]. Therein, the $\mathrm{XC}$ energy is generally expressed as $E_{X C}=E_{X}^{G G A}+$ $E_{C}^{L D A}+E_{C}^{\text {non-local }}$, where $E_{X}^{G G A}$ is the exchange term from a given GGA $\mathrm{XC}$ functional. The local correlation energy $E_{C}^{L D A}$, however, is identical for most vdW functionals. By definition, the nonlocal part of the correlation energy $E_{C}^{\text {non-local }}$ does not suffer from the Coulomb self-energy of each electron. Hence, XC self-interaction errors in vdW-DF schemes are mostly related to the corresponding exchange part. Similar to semilocal GGA XC functionals, the results of vdW-DF approaches depend on the employed $E_{X}^{G G A}$ [15-17]. There are many noncovalent systems, where the accuracy of DFT falls short of requirements, particularly if the problem is to distinguish between molecular crystal phases and competing low-energy polymorphs. As a simple molecular system, the energy differences between crystalline benzene and its polymorphs under pressure are less than a few kilojoules per mole. It has been demonstrated that the use of ab initio many-electron wave-function methods is essential to tackle this problem [18].

Interestingly, the similarity between Schrödinger's equation in imaginary time and the diffusion equation suggests employing a stochastic diffusion-based process for solving the manybody Schrödinger equation [19-21]. In fact, quantum Monte Carlo (QMC) [22-24], which is a family of stochastic methods for solving the Schrödinger equation, is becoming an effective approach for investigating vdW interactions [15,25,26]. In particular, previous studies have shown that diffusion Monte Carlo (DMC) can provide accurate energies for atoms [27], molecules [28], and crystals [29-34] with noncovalent interactions $[16,35,36]$.

In order to mimic an extended system, QMC simulations of crystals are performed using finite simulation cells subject to periodic boundary conditions. Yet practical and computational 
constraints restrict the maximum size of the simulation cell and so introduce finite-size (FS) errors, which can be rather large. The FS effects are larger in QMC than in mean-field methods because electrons are explicitly represented. Quantifying and minimizing these errors is an essential part of all QMC simulations of extended systems, particularly when high accuracy is required.

In this work, we employ variational Monte Carlo (VMC) [37], as well as DMC [19], to study the Coulomb interaction between benzene rings in solid form with $\mathrm{Pbca}$ symmetry. Crystalline benzene, due to its aromatic vdW interactions, is a model structure for studying noncovalent interactions in solids. Specifically, we calculate the vdW energy between four benzene molecules in a periodic simulation cell. We use the fragment-based approach, where the only degree of freedom is the distance between the center of mass of the benzene molecules.

The energy gap of crystalline benzene has attracted a considerable amount of interest because of its importance in fundamental and applied science [38]. The energy gap $E_{g}$ is defined as the difference between the ionization energy and electron affinity. The Coulomb interactions between molecules, which are packed in a crystalline phase, reduce the fundamental gap compared to the gas phase. This renormalized energy gap effect is crucial in organic electronics, especially in charge transport. The gas-phase energy gap is typically several electron volts, which is rather large for practical applications. However, DFT-based methods are unable to quantify the fundamental energy gap of solid benzene and to distinguish the gas-phase gap from that of the crystallized structure $[39,40]$. Yet the energy band gap of a molecular crystal, as determined by the GW approximation to many-body perturbation theory, is in agreement with experimental measurements [41,42].

We employ the DMC approach to accurately calculate the excitonic energy gap of crystalline benzene. Although the DMC method was initially developed to study only groundstate properties, it can also be applied to determine excitedstate spectra in atoms, molecules, and crystals [32,43,44]. The excitonic energy gap is smaller than the quasiparticle band gap, which is usually determined by the GW approximation. The reason is the attraction between the excited electron in the conduction band and the introduced hole in the valence band. The exciton binding energy is defined as the difference between the quasiparticle and the excitonic energy gaps. When an electron is added to (removed from) a finite simulation cell with periodic boundary conditions, a periodic lattice of quasiparticles (quasiholes) is created. The energy of this lattice of quasiparticles is similar to the Madelung constant of the simulation cell lattice and introduces a large FS error in the electron affinity and ionization potential. The FS error in the quasiparticle energy gap is much larger than the excitonic gap for which the number of electrons is fixed. When an electron is added to (removed from) a finite and periodic simulation cell in which the interaction between particles is controlled by the Ewald potential, a neutralizing charge-density background is applied which vanishes in the infinite-system-size limit. Hence, the quasiparticle band gap can be physically meaningful in the infinite-system-size limit.

The remainder of this paper is organized as follows. Section II describes the details of our VMC and DMC calculations.
The corresponding results are discussed in Sec. III, which is followed by our conclusions in Sec. IV.

\section{COMPUTATIONAL DETAILS}

The DMC method is a stochastic technique for calculating the zero-temperature total electronic energy of a many-electron system [19]. Even though DMC has been described in previous review articles [22-24], we will nevertheless start with a brief explanation of the general scheme since there are some technical aspects in this work we feel are rather important.

More precisely, the DMC method solves the imaginary-time Schrödinger equation

$$
\frac{\partial \Psi(\mathbf{R}, \tau)}{\partial \tau}=\frac{1}{2} \sum_{i=1}^{N_{e}} \nabla_{\mathbf{r}_{i}}^{2} \Psi(\mathbf{R}, \tau)-\left[V(\mathbf{R})-E_{T}\right] \Psi(\mathbf{R}, \tau),
$$

where $\mathbf{R}=\left(\mathbf{r}_{1}, \mathbf{r}_{2}, \ldots, \mathbf{r}_{N_{e}}\right)$ is a $3 N_{e}$-dimensional vector representing the positions of all $N_{e}$ electrons in the simulation cell, $\tau$ is the imaginary time, $V(\mathbf{R})$ is the potential energy including electron-electron interactions, and $E_{T}$ is a constant energy offset. Throughout, Hartree atomic units are assumed; that is, the numerical values of $\hbar, e, m_{e}$, and $4 \pi \varepsilon_{0}$ are all identical to 1 . As already alluded to above, the imaginary-time Schrödinger equation is similar to a $3 N_{e}$-dimensional diffusion equation with diffusion constant $D=1 / 2$. The potentialenergy term causes the diffusers to "branch" (multiply or die out) at a position-dependent rate. The wave function $\Psi(\mathbf{R}, \tau)$ is the number density of diffusers, which are normally known as walkers or configurations and are points in the $3 N_{e^{-}}$ dimensional configuration space, not individual electrons. The DMC method employs this physical interpretation to simulate the imaginary-time evolution of the wave function using a finite population of diffusing and branching walkers.

By solving the imaginary-time Schrödinger equation, the electronic ground-state is projected out as $\tau \rightarrow \infty$. If the initial wave function is expanded as a linear combination of energy eigenfunctions $\Psi(\tau=0)=\sum_{i} c_{i} \Psi_{i}$, the solution of the imaginary-time Schrödinger equation $\partial \Psi / \partial \tau=-(\hat{H}-$ $\left.E_{T}\right) \Psi$ is

$$
\Psi(\tau)=\sum_{i} c_{i} e^{-\left(E_{i}-E_{T}\right) \tau} \Psi_{i}
$$

Thus, as long as $c_{0} \neq 0$, the wave function $\Psi(\tau)$ becomes proportional to $\Psi_{0}$ as $\tau \rightarrow \infty$. By gradually adjusting $E_{T}$ to maintain the normalization of the solution in the large- $\tau$ limit, we can find the ground-state energy $E_{0}$.

Nevertheless, a fundamental difficulty with this approach is that the wave function $\Psi(\mathbf{R}, \tau)$, which is not necessarily positive, is interpreted as a walker density that must be positive by its very definition. The naive application of the DMC algorithm to a many-electron system yields a totally symmetric many-boson ground-state of no physical interest. The so-called fixed-node approximation requires a trial wave function $\Psi_{T}(\mathbf{R})$, which imposes a fixed nodal constraint and hence prevents walker moves that cause $\Psi_{T}$ to change sign. As long as $\Psi_{T}$ is properly antisymmetric, this is sufficient to ensure that a fermionic solution is obtained. It can be shown that the energies calculated within the fixed-node approximation are variational [22]: the result is greater than or equal to the 
many-fermion ground-state energy and tends to the exact energy as the $\left(3 N_{e}-1\right)$-dimensional nodal surface, on which $\Psi_{T}=0$, approaches the ground-state nodal surface. However, assuming the fixed-node approximation is essential for DMC simulations of large systems, it is the only fundamental limitation of the method. Other approximations, such as the use of a finite time step or the representation of ions by pseudopotentials, can be made negligible or fully avoided given sufficient computer time.

The diffusion and branching process described above is unstable in practice since the potential energy $V(\mathbf{R})$ diverges whenever electrons approaches nuclei or each other, leading to an uncontrollable branching. This problem, however, can be overcome using an importance-sampling technique. To that extent, the imaginary-time Schrödinger equation is rewritten in terms of the quantity $f(\mathbf{R}, \tau)=\Psi_{T}(\mathbf{R}) \Psi(\mathbf{R}, \tau)$ to obtain

$$
\begin{aligned}
\frac{\partial f(\mathbf{R}, \tau)}{\partial t}= & \frac{1}{2} \nabla_{\mathbf{R}}^{2} f(\mathbf{R}, \tau)-\nabla_{\mathbf{R}} \cdot[\mathbf{v}(\mathbf{R}) f(\mathbf{R}, \tau)] \\
& -\left[E_{L}(\mathbf{R})-E_{T}\right] f(\mathbf{R}, \tau),
\end{aligned}
$$

where $\nabla_{\mathbf{R}}=\left(\nabla_{\mathbf{r}_{1}}, \nabla_{\mathbf{r}_{2}}, \ldots, \nabla_{\mathbf{r}_{N_{e}}}\right)$ is the $3 N_{e}$-dimensional gradient operator, $\nabla_{\mathbf{R}}^{2}=\nabla_{\mathbf{R}} \cdot \nabla_{\mathbf{R}}$ is the corresponding Laplacian, $\mathbf{v}(\mathbf{R})=\nabla_{\mathbf{R}} \ln \left|\Psi_{T}(\mathbf{R})\right|$ is the $3 N_{e}$-dimensional drift-velocity vector, and $E_{L}(\mathbf{R})=\left[1 / \Psi_{T}(\mathbf{R})\right] \hat{H} \Psi_{T}(\mathbf{R})$ is the local energy. The importance-sampled imaginary-time Schrödinger equation corresponds to a diffusion process similar to that discussed above, except that the walkers now drift with velocity $\mathbf{v}(\mathbf{R})$, as well as diffusing and branching. The branching rate is determined by the shifted local energy $E_{L}(\mathbf{R})-E_{T}$ instead of the shifted potential energy $V(\mathbf{R})-E_{T}$. If the trial function is a good approximation to the ground state, the local energy is a smooth function of $\mathbf{R}$, and the numerical difficulties caused by divergences in $V(\mathbf{R})$ are bypassed. The fixed-node approximation is imposed by rejecting walker moves that change the sign of $\Psi_{T}(\mathbf{R})$.

In this work, the CASINO code [45] was used to perform DMC simulations with a trial wave function of the SlaterJastrow (SJ) form,

$$
\Psi_{\mathrm{SJ}}(\mathbf{R})=\exp [J(\mathbf{R})] \operatorname{det}\left[\psi_{n}\left(\mathbf{r}_{i}^{\uparrow}\right)\right] \operatorname{det}\left[\psi_{n}\left(\mathbf{r}_{j}^{\downarrow}\right)\right],
$$

where $\mathbf{R}$ is a $3 N$-dimensional vector containing the positions of all $N$ electrons, $\mathbf{r}_{i}^{\uparrow}$ is the position of the $i$ th spin-up electron, $\mathbf{r}_{j}^{\downarrow}$ is the position of the $j$ th spin-down electron, $\exp [J(\mathbf{R})]$ is the Jastrow correlation factor, and $\operatorname{det}\left[\psi_{n}\left(\mathbf{r}_{i}^{\uparrow}\right)\right]$ and $\operatorname{det}\left[\psi_{n}\left(\mathbf{r}_{j}^{\downarrow}\right)\right]$ are Slater determinants made of spin-up and spin-down one-electron wave functions. These orbitals were obtained from Perdew-Burke-Ernzerhof (PBE) plus DFT calculations performed with the CASTEP plane-wave code [46] in conjunction with Trail-Needs Dirac-Fock pseudopotentials $[47,48]$. For the purpose of approaching the complete-basis-set limit [49], a large energy cutoff of $4000 \mathrm{eV}$ was chosen. The resulting plane-wave orbitals were subsequently transformed into a localized blip polynomial basis [50]. Our DMC results were obtained using a real $\Gamma$-point wave function.

The Jastrow correlation factor in Eq. (5) is a positive, symmetric, explicit function of interparticle distances in the form of

$$
\begin{aligned}
J\left(\mathbf{r}_{i}, \mathbf{r}_{I}\right)= & \sum_{I=1}^{M} \sum_{i=1}^{N} u_{1}\left(\mathbf{r}_{i I}\right)+\sum_{i=1}^{N-1} \sum_{j=i+1}^{N} u_{2}\left(\mathbf{r}_{i j}\right) \\
& +\sum_{I=1}^{M} \sum_{i=1}^{N-1} \sum_{j=i+1}^{N} u_{3}\left(\mathbf{r}_{i I}, \mathbf{r}_{j I}, \mathbf{r}_{i j}\right),
\end{aligned}
$$

where $N, M, \mathbf{r}_{i}$, and $\mathbf{r}_{I}$ are the number of electrons, number of ions, the position of electron $i$, and the position of nucleus $I$, whereas $\mathbf{r}_{i j}=\mathbf{r}_{i}-\mathbf{r}_{j}$ and $\mathbf{r}_{i I}=\mathbf{r}_{i}-\mathbf{r}_{I}$. The polynomial one-body electron-nucleus (1b), two-body electron-electron (2b), and three-body electron-electron-nucleus ( $3 \mathrm{~b})$ terms are denoted as $u_{1}\left(r_{i I}\right), u_{2}\left(r_{i j}\right)$, and $u_{3}\left(r_{i I}, r_{j I}, r_{i j}\right)$, respectively.

We also studied the contribution of nondynamical correlation by including the inhomogeneous backflow (BF) coordinate transformation into the SJ wave function [51]. Our BF transformation includes an electron-electron correlation factor as well as electron-proton terms and is given by

$$
\mathbf{X}_{i}\left(\left\{\mathbf{r}_{j}\right\}\right)=\mathbf{r}_{i}+\boldsymbol{\xi}_{i}^{(e-e)}\left(\left\{\mathbf{r}_{j}\right\}\right)+\boldsymbol{\xi}_{i}^{(e-P)}\left(\left\{\mathbf{r}_{j}\right\}\right),
$$

where $\mathbf{X}_{i}\left(\left\{\mathbf{r}_{j}\right\}\right)$ is the transformed coordinate of electron $i$, which depends on the full configuration of the system $\left\{\mathbf{r}_{j}\right\}$. The vector functions $\boldsymbol{\xi}_{i}^{(e-e)}\left(\left\{\mathbf{r}_{j}\right\}\right)$ and $\boldsymbol{\xi}_{i}^{(e-P)}\left(\left\{\mathbf{r}_{j}\right\}\right)$ are the electron-electron and electron-proton backflow displacements of electron $i$. They are parameterized as

$$
\boldsymbol{\xi}_{i}^{(e-e)}\left(\left\{\mathbf{r}_{j}\right\}\right)=\sum_{j \neq i}^{N_{e}} \alpha_{i j}\left(r_{i j}\right) \mathbf{r}_{i j}
$$

and

$$
\boldsymbol{\xi}_{i}^{(e-P)}\left(\left\{\mathbf{r}_{j}\right\}\right)=\sum_{I}^{N_{P}} \beta_{i I}\left(r_{i I}\right) \mathbf{r}_{i I},
$$

where $\alpha_{i j}\left(r_{i j}\right)$ and $\beta_{i I}\left(r_{i I}\right)$ are polynomial functions of electron-electron and electron-proton distances, respectively, and contain variational parameters. In this way, the resulting backflow SJ (BSJ) is able to adapt the nodal surface in order to recover the static correlation energy that is characteristic for multireference systems [52]. All adjustable parameters in the Jastrow correlation factor and $\mathrm{BF}$ coordinate transformation are optimized by minimizing the variance, as well as the variational energy at the VMC level [53,54]. The Kato cusp conditions are enforced so that the local energy is finite when two electrons or an electron and a nucleus are coincident $[55,56]$. Specifically, the electron-electron cusp conditions are imposed on the parameters of the Jastrow correlation factor, and the electron-nucleus ones are imposed on the orbitals within the Slater determinant. Since the backflow coordinate transformation can modify the cusp conditions, we have constrained the backflow parameters so that they are not [51].

The excitonic energy band gap is determined by promoting an electron from a valence-band state into a conduction-band orbital at the $\Gamma$ point. The singlet excited state was defined by promoting an electron without flipping its spin:

$$
\Delta_{\mathrm{exc}}=E_{1}-E_{0},
$$



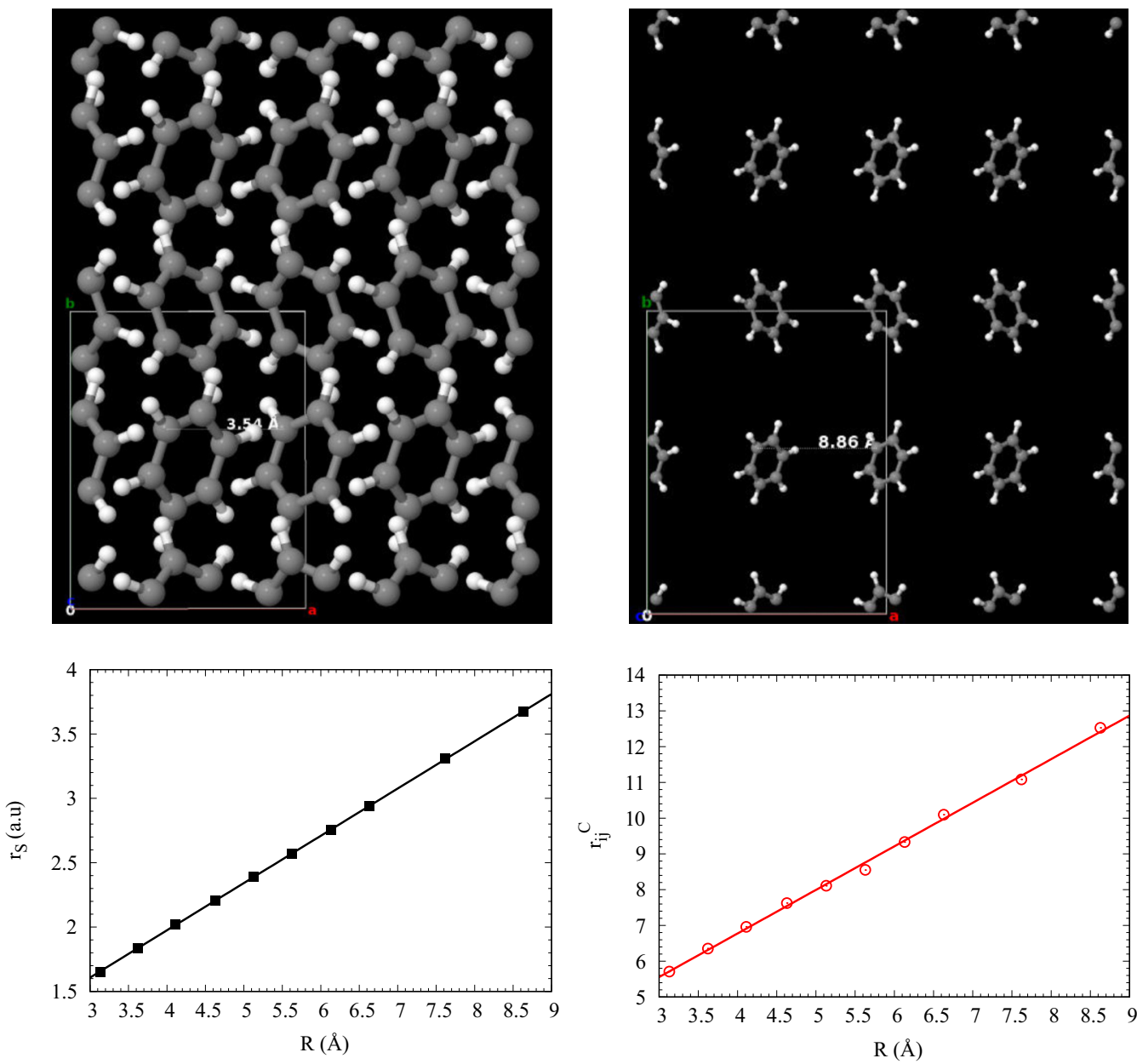

FIG. 1. Top: The smallest and largest simulation cells with volumes $V_{s}=336.4334 \AA^{3}$ and $V_{l}=3691.9989 \AA^{3}$, respectively, which are used to calculate the vdW interactions between the benzene molecules. Bottom: Wigner-Seitz radius $r_{S}$ and cutoff length $r_{i j}^{C}$ as linear functions of $R$.

where $E_{0}$ and $E_{1}$ are the DMC energies of the ground and excitonic states, respectively. The excitonic energy equals the vertical optical absorption gap.

The FS errors are categorized into one- (independent particle) and many-body terms. The one-body term includes the noninteracting kinetic, potential, and Hartree energies. The one-body FS errors are much more important in metallic systems due to shell-filling effects [57]. The single-particle errors in metallic systems are eliminated using canonical and grand-canonical twist-averaging boundary conditions [58,59]. Many-body FS errors are due to the exchange and correlation effects within the Coulomb and kinetic energies and cannot be removed by twist averaging. Crystalline benzene is a wide-band-gap insulator, which is why many-body FS effects are the main source of errors in our DMC calculations. Hence, we investigate the influence of these FS errors on the $\mathrm{vdW}$ interaction between aromatic benzene rings. There are different approaches to reduce or cancel many-body errors. The most widely used and perhaps oldest approximation is extrapolating to the infinite-system-size limit, which, however, is computationally rather expensive for the large simulation cells that are studied here. We therefore analyze two alternative methods to correct for many-body FS errors in our DMC simulations. Specifically, we apply the structure-factor-based approaches proposed by Chiesa et al. [60], which allows us to estimate the effect of many-body FS errors in the potential and kinetic energies based on the random-phase approximation. The main assumption in this approach is that the low- $k$ behavior of the structure factor is independent of the shape of the simulation cell. Here, we first apply the standard Ewald form of the periodic Coulomb interaction and Chiesa et al.'s FS corrections for both the kinetic $(\triangle \mathrm{KE})$ and potential $(\triangle \mathrm{PE})$ energies. Second, we employ the model-periodic Coulomb (MPC) interactions $[61,62]$ to deal with the Coulomb errors. Our DMC results obtained with both of these approaches are expected to be similar.

\section{RESULTS AND DISCUSSION}

The orthorhombic simulation cell with Pbca symmetry contains four benzene molecules and 120 electrons. More precisely, we are considering ten different simulation cell sizes, whose volumes are between $V_{s}=336.4334 \AA^{3}$ and $V_{l}=$ $3691.9989 \AA^{3}$, respectively. The volume of the simulation cells was varied in such a way that the distance $R$ between the center of mass of the benzene molecules is a linear function of $r_{S}$ (electronic Wigner-Seitz radius), as shown in Fig. 1. 


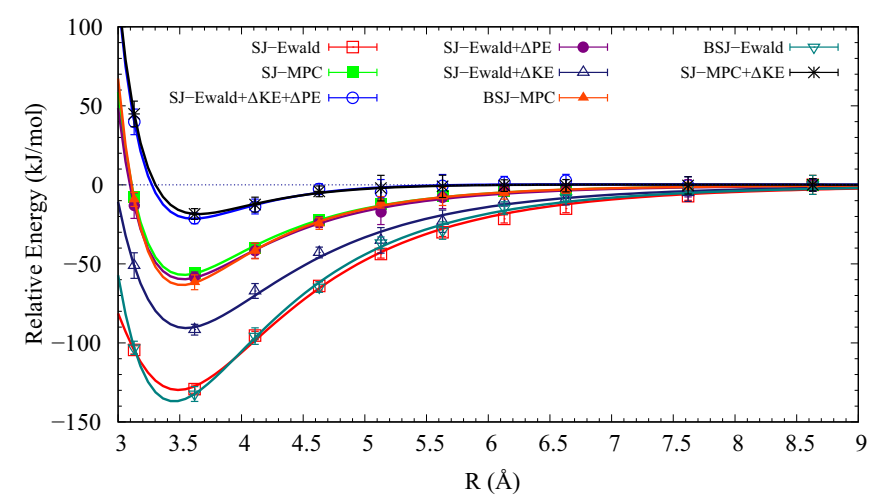

FIG. 2. Relative DMC energy as calculated using the Ewald and MPC potentials and SJ and BSJ wave functions. The data points are fitted to $\frac{C_{6}}{R^{6}}+\frac{C_{8}}{R^{8}}+\frac{C_{10}}{R^{10}}$, where $C_{n}$, with $n=6,8,10, \ldots$, are fitting parameters and $R$ is the smallest distance between the center of mass of the benzene molecules. The reference line is the DMC energy at the largest separation.

The relative configurations of the benzene molecules and their geometries were fixed.

In total, there are 151 and 225 variational parameters in our SJ and BSJ wave functions, respectively. We first optimized the expansion coefficients of the Jastrow correlation factor of the SJ wave function. The optimized coefficients were subsequently used to also optimize cutoff length in the Jastrow term. The optimized Jastrow term was reused to generate the BSJ wave function. In our BSJ wave-function optimization, we first optimized the variational parameters of the BF coordinate transformation while the parameters in the Jastrow correlation factor were kept fixed before we reoptimized all the variational parameters together. We found that the described optimization procedure produces an accurate wave function for DMC calculations. The two-body $u_{2}\left(\mathbf{r}_{i j}\right)$ term consists of a power expansion in $r_{i j}$ and goes to zero at the cutoff length $r_{i j}^{C}$. We found that our optimized values for $r_{i j}^{C}$ represent essentially a linear function of $R$.

The resulting nonlocal vdW energy curves between four benzene molecules, which are calculated using the Ewald and MPC interactions, are illustrated in Fig. 2. The DMC data points are reported in the Supplementary Material [63]. An advantage of the MPC approach is that it reduces FS errors arising from the use of the Ewald interaction. As an alternative to the MPC scheme, many-body contributions to the FS errors can also be minimized using the FS correction to the exchange-correlation $(\triangle \mathrm{PE})$ and kinetic $(\triangle \mathrm{KE})$ energies [60]. The total energy of the system at the largest separation is considered as the zero reference. We would like to emphasize that in our approach, the size-consistency problem within binding-energy calculations [64] is avoided. The results of the BSJ wave function with the Ewald and MPC interactions are also shown in Fig. 2. As can be seen, employing the MPC interaction corrects the Coulomb FS errors but not the manybody FS error of the kinetic energy. Also, the binding-energy curve, as calculated by the SJ-Ewald $+\triangle \mathrm{PE}$ scheme, agrees well with the corresponding SJ-MPC results. Even though the magnitude of $\triangle \mathrm{KE}$ is smaller than that of $\triangle \mathrm{PE}$, combining both FS correction techniques entails the largest contribution.
TABLE I. The cohesive energy of crystalline benzene as obtained by different DMC approaches at $0 \mathrm{~K}$. All energies are in $\mathrm{kJ} / \mathrm{mol}$. The quantum-mechanical zero-point energy contribution, which amounts to $2.8 \mathrm{~kJ} / \mathrm{mol}$ [65], is not included in our results. DMC results are compared with the cohesive energies which are obtained using coupled cluster single double (Triple, Quadrapole) (CCSD(T,Q)) and complete basis set (CBS); hybrid functional of Becke (B) 3-parameter Lee-Yang-Parr (LYP) combined with the Grimme empirical model (B3LYP-D Grimme) and with the standard basis set of 6-31G(d,p) and triple zeta with 1 polarization function (TZP); density functional theory (DFT) with local density approximation (LDA); exact exchange (EXX); random phase approximation (RPA); and PerdewBurke-Ernzerhof (PBE) functional.

\begin{tabular}{|c|c|c|}
\hline Approach & Energy & Reference \\
\hline DMC SJ Ewald & $-125.4 \pm 9.6 \mathrm{t}$ & this work \\
\hline DMC SJ MPC & $-57.9 \pm 9.6$ & this work \\
\hline DMC SJ Ewald $+\Delta \mathrm{PE}$ & $-57.9 \pm 9.6$ & this work \\
\hline DMC SJ Ewald $+\Delta \mathrm{KE}$ & $-86.8 \pm 9.6$ & this work \\
\hline DMC SJ Ewald $+\Delta \mathrm{PE}+\Delta \mathrm{KE}$ & $-29.0 \pm 9.6$ & this work \\
\hline $\mathrm{DMC} S \mathrm{SJ} \mathrm{MPC}+\Delta \mathrm{KE}$ & $-29.0 \pm 9.6$ & this work \\
\hline DMC BSJ Ewald & $-135.1 \pm 9.6 \mathrm{t}$ & this work \\
\hline DMC BSJ MPC & $-67.5 \pm 9.6$ & this work \\
\hline DMC SJ MPC & $-52.1 \pm 0.4$ & {$[36]$} \\
\hline Estimated experiment at $0 \mathrm{~K}$ & $-55.3 \pm 2.2$ & {$[18]$} \\
\hline $\begin{array}{l}\text { Quantum chemistry [coupled cluster } \\
\text { single double (Triple, Quadrapole) } \\
\text { (CCSD(T,Q))] }\end{array}$ & $-55.9 \pm 0.76 \pm 0.1$ & $1[18]$ \\
\hline $\begin{array}{l}\text { Hybrid functional of Becke (B) } \\
\text { 3-parameter Lee-Yang-Parr (LYP) } \\
\text { combined with the Grimme empirical } \\
\text { model (B3LYP-D Grimme) and with } \\
\text { the standard basis set of 6-31G(d,p) }\end{array}$ & $-48.2 \pm 20.1$ & [66] \\
\hline $\begin{array}{l}\text { B3LYP-D Grimme/triple zeta with } 1 \\
\text { polarization function (TZP) }\end{array}$ & $-46.5 \pm 1.9$ & {$[66]$} \\
\hline B3LYP/6-31G(d,p) & $-5.8 \pm 8.9$ & [66] \\
\hline $\mathrm{CCSD}(\mathrm{T}) /$ complete basis set $(\mathrm{CBS})$ & -56.4 & {$[67,68]$} \\
\hline Typical experimental values & -43 to -47 & {$[69]$} \\
\hline $\begin{array}{l}\text { Density functional theory (DFT) with } \\
\text { local density approximation (LDA) }\end{array}$ & -57.00 & [70] \\
\hline $\begin{array}{l}\text { Exact exchange (EXX)/random phase } \\
\text { approximation (RPA) (LDA) }\end{array}$ & -44.00 & [70] \\
\hline $\begin{array}{l}\text { DFT Perdew-Burke-Ernzerhof (PBE) } \\
\text { functional }\end{array}$ & -9.60 & [70] \\
\hline EXX/RPA (PBE) & -47.00 & {$[70]$} \\
\hline $\mathrm{DFT} / \mathrm{LDA}+\mathrm{B}$ & -34 & [71] \\
\hline $\mathrm{DFT} / \mathrm{LDA}+\mathrm{B}+\mathrm{LYP}$ & -65 & [71] \\
\hline
\end{tabular}

Interestingly, the results using the SJ and BSJ wave functions are remarkably similar to each other. The Ewlad $+\Delta \mathrm{KE}+\Delta \mathrm{PE}$ results are in excellent agreement with the DMC energies which are calculated using MPC $+\triangle \mathrm{KE}$. Note that the higherorder kinetic-energy corrections defined according to Eq. (55) in Ref. [57] are included in $\triangle \mathrm{KE}$. The DMC energy curves were all fitted to $\frac{C_{6}}{R^{6}}+\frac{C_{8}}{R^{8}}+\frac{C_{10}}{R^{10}}$, where $C_{n}$, with $n=6,8,10, \ldots$, are fitting parameters and $R$ is the smallest distance between the center of mass of the benzene molecules.

We computed the binding energy between the aromatic rings, which indicates the strength of the vdW forces holding the benzene molecules together. The cohesive energy $E_{\text {coh }}$ is 


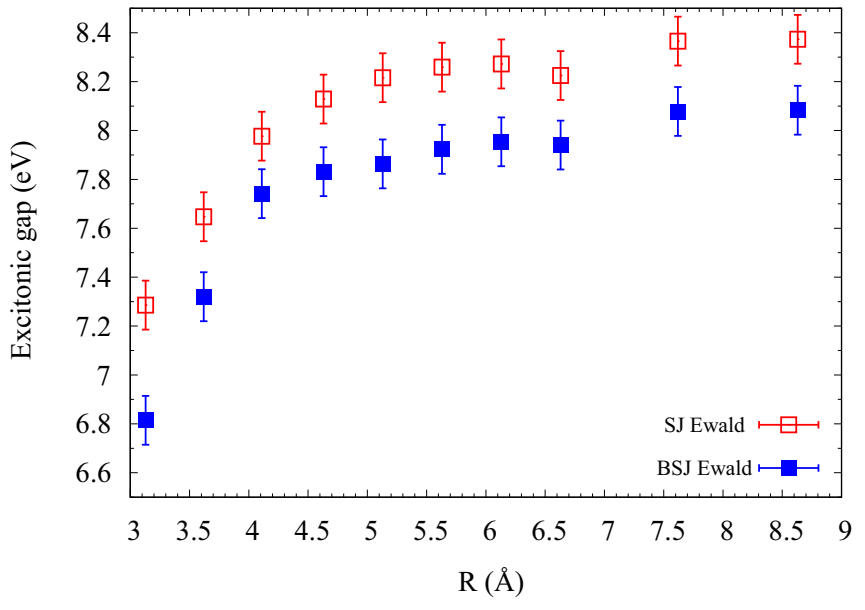

FIG. 3. Singlet DMC excitonic gap, which is determined using the SJ and BSJ wave functions in conjunction with the bare Ewald potential as a function of $R$.

defined as $E_{\mathrm{coh}}=E_{D M C}^{R_{0}}-E_{D M C}^{R_{\infty}}$, where $E_{D M C}^{R_{0}}$ and $E_{D M C}^{R_{\infty}}$ are the DMC energies of the system at the equilibrium distance $R_{0}$ and infinite separation $R_{\infty}$, respectively. For the latter, we assume that $R_{\infty}=8.64 \AA$. Our cohesive energies, as determined using different DMC schemes, are listed in Table I. We find that using the bare Ewald interaction, the cohesive energy is severely overestimated due to the presence of significant FS errors. However, correcting for the latter by including $\triangle \mathrm{PE}$ and $\triangle \mathrm{KE}$, the cohesive energy is reduced by as much as $96 \mathrm{~kJ} / \mathrm{mol}$. Moreover, comparing the contributions of $\triangle \mathrm{PE}$ and $\triangle \mathrm{KE}$ immediately suggests that the former is more effective and outperforms the $\Delta \mathrm{KE}$ FS correction by $29 \mathrm{~kJ} / \mathrm{mol}$. In fact, the $\triangle \mathrm{PE} F S$ correction is equally effective as employing the MPC approach. Although including the BF coordinate transformation generally improves the ground-state total energy, the cohesive energies, as obtained by the SJ Ewald and BSJ Ewald schemes, differ by just 9.5(1) kJ/mol, which is also the case when adding the MPC interaction. That is, when calculating energy differences using QMC, the impact of FS errors is particularly important and may even be more important than the particular trial wave function. Recently, the DMC method was applied to calculate the equation of state of molecular crystals [36]. In particular, the authors calculated the lattice energy of crystalline benzene using $E_{\text {latt }}=E_{\text {crys }}-E_{\text {gas }}$, which is listed in Table I. Our results obtained using the MPC potential agree well with those recently reported ones.

The singlet DMC excitonic gaps, which were obtained at different densities using the SJ and BSJ wave functions, are shown in Fig. 3. The highest density (smallest $R$ ) corresponds to crystalline benzene at $8 \mathrm{GPa}$ pressure, while the lowest density (largest $R$ ) mimics the gas phase. On the one hand, the energy gaps computed by means of HF theory are typically too large due to the absence of electron-electron correlation. On the other hand, the band gaps calculated with conventional DFT methods are generally too small. In DMC simulations, however, multiplying the Slater determinant made up of HF or DFT orbitals by a Jastrow correlation factor permits us to retrieve a large amount of the correlation energy and results in energy gaps much closer to experiment. But applying the Jastrow correlation factor does not alter the nodal surface of the trial wave function, which is determined by the Slater determinant. Nevertheless, introducing a BF coordinate transformation to the SJ wave function affects the nodal surface and improves the ability of DMC to recover nondynamical correlation energy. As we found, adding a BF coordinate transformation lowers the excitonic energy gap. When calculated with the GW approximation, the corresponding highest occupied and lowest unoccupied molecular orbital gaps of benzene in the gas and crystal phases are 10.51 and $7.91 \mathrm{eV}$, respectively [42]. By contrast, at the DFT-LDA level of theory, the electron addition and removal energies of benzene in the gas and crystal phases are 5.16 and $5.07 \mathrm{eV}$, respectively. For comparison, the experimental ionization potential of a benzene molecule is $9.25 \mathrm{eV}$ [72]. Using modified hybrid and constrained DFT calculations $[39,40]$, the fundamental gap renormalization was previously found to be about $\sim 2 \mathrm{eV}$. The SJ-DMC calculations predict that the energy gaps at optimized and largest $R$ are 7.7(1) and 8.4(1) eV, respectively. The energy gaps which are obtained using BSJ-DMC simulations are 7.4(1) and 8.1(1) $\mathrm{eV}$, respectively. We calculated the gap renormalization using the difference between energy gaps at optimized $R$ and the largest $R$. Hence, DMC excitonic energy gap results yield a gap renormalization of $0.7(1) \mathrm{eV}$.

However, DMC simulations of excitations spectra in solids are rather challenging due to a $1 / N$ effect: the change in the total energy induced by a one- or two-particle excitation is inversely proportional to the number of electrons in the simulation cell. Thus, generally, a relatively large simulation cell is essential for a high-precision description of the infinite crystal. Yet our dispersion energy curves demonstrate the importance of explicitly accounting for FS errors, in which case the interactions between benzene molecules are rather accurately described.

\section{CONCLUSIONS}

In this work, the nonlocal vdW interactions between benzene molecules was studied by means of DMC simulations using the SJ and BSJ wave functions. We found that, when calculating energy differences, the results are much more affected by FS errors than generally appreciated. In the case of the cohesive energy, FS errors can be as large as $96 \mathrm{~kJ} / \mathrm{mol}$, which is much more pronounced than the impact of the BF coordinate transformation to include nondynamical correlation effects. In addition, we also calculated the singlet excitonic energy gap for benzene in the gas and solid phases. At variance with the cohesive energy, the inclusion of BF in the trial wave function entails a reduction of the excitonic band gap. Eventually, we also obtained a high-accuracy estimation of the benzene gap renormalization.

\section{ACKNOWLEDGMENTS}

The authors would like to thank the Gauss Center for Supercomputing (GCS) for providing computing time through the John von Neumann Institute for Computing (NIC) on the GCS share of the supercomputer JUQUEEN at the Jülich Super- 
computing Centre (JSC). Additional computing facilities were provided through the DECI-13 PRACE project QMCBENZ15 and the Dutch national supercomputer Cartesius. This project has received funding from the European Research Council (ERC) under the European Union's Horizon 2020 research and innovation program (Grant Agreement No. 716142).
[1] F. London, Z. Phys. Chem., Abt. B 11, 222 (1930).

[2] A. Koide, J. Phys. B 9, 3173 (1976).

[3] K. Tang and J. P. Toennies, J. Chem. Phys. 80, 3726 (1984).

[4] J. Hepburn and G. Scoles, Chem. Phys. Lett. 36, 451 (1975).

[5] R. Ahlrichs, R. Penco, and G. Scoles, Chem. Phys. 19, 119 (1977).

[6] X. Wu, M. C. Vargas, S. Nayak, V. Lotrich, and G. Scoles, J. Chem. Phys. 115, 8748 (2001).

[7] U. Zimmerli, M. Parrinello, and P. Koumoutsakos, J. Chem. Phys. 120, 2693 (2004).

[8] E. R. Johnson and A. D. Becke, J. Chem. Phys. 123, 024101 (2005).

[9] S. Grimme, J. Comput. Chem. 25, 1463 (2004); 27, 1787 (2006); 18, 9955 (2012).

[10] A. Tkatchenko and M. Scheffler, Phys. Rev. Lett. 102, 073005 (2009).

[11] L. M. Woods, D. A. R. Dalvit, A. Tkatchenko, P. RodriguezLopez, A. W. Rodriguez, and R. Podgornik, Rev. Mod. Phys. 88, 045003 (2016).

[12] M. Dion, H. Rydberg, E. Schröder, D. C. Langreth, and B. I. Lundqvist, Phys. Rev. Lett. 92, 246401 (2004)

[13] V. R. Cooper, Phys. Rev. B 81, 161104 (2010)

[14] K. Lee, E. D. Murray, L. Kong, B. I. Lundqvist, and D. C. Langreth, Phys. Rev. B 82, 081101(R) (2010).

[15] S. Azadi and R. E. Cohen, J. Chem. Phys. 145, 064501 (2016).

[16] S. Azadi and G. J. Ackland, Phys. Chem. Chem. Phys. 19, 21829 (2017).

[17] R. Podeszwa, B. M. Rice, and K. Szalewicz, Phys. Rev. Lett. 101, 115503 (2008).

[18] J. Yang, W. Hu, D. Usvyat, D. Matthews, M. Schutz, and G. K.-L. Chan, Science 345, 640 (2014).

[19] D. M. Ceperley and B. J. Alder, Phys. Rev. Lett. 45, 566 (1980).

[20] D. M. Ceperley and B. J. Alder, J. Chem. Phys. 81, 5833 (1984).

[21] P. J. Reynolds, D. M. Ceperley, B. J. Alder, and W. A. Lester, Jr., J. Chem. Phys. 77, 5593 (1982).

[22] W. M. C. Foulkes, L. Mitas, R. J. Needs, and G. Rajagopal, Rev. Mod. Phys. 73, 33 (2001).

[23] J. Kolorenc and L. Mitas, Rep. Prog. Phys. 74, 026502 (2011).

[24] A. Lüchow, Wiley Interdiscip. Rev. Comput. Mol. Sci. 1, 388 (2011).

[25] S. Azadi and R. E. Cohen, J. Chem. Phys. 143, 104301 (2015).

[26] M. Dubecký, L. Mitas, and P. Jurečka, Chem. Rev. 116, 5188 (2016).

[27] M. Marchi, S. Azadi, M. Casula, and S. Sorella, J. Chem. Phys. 131, 154116 (2009).

[28] S. Azadi, R. Singh, and T. D. Kühne, Int. J. Quantum Chem. 115, 1673 (2015).

[29] M. Marchi, S. Azadi, and S. Sorella, Phys. Rev. Lett. 107, 086807 (2011).

[30] S. Azadi and W. M. C. Foulkes, J. Chem. Phys. 143, 102807 (2015).

[31] S. Azadi, B. Monserrat, W. M. C. Foulkes, and R. J. Needs, Phys. Rev. Lett. 112, 165501 (2014).
[32] S. Azadi, N. D. Drummond, and W. M. C. Foulkes, Phys. Rev. B 95, 035142 (2017).

[33] S. Azadi and T. D. Kühne, J. Chem. Phys. 146, 084503 (2017).

[34] S. Azadi, W. M. C. Foulkes, and T. D. Kühne, New J. Phys. 15, 113005 (2013).

[35] J. Řezáč and P. Hobza, Chem. Rev. 116, 5038 (2016).

[36] A. Zen, J. G. Brandenburg, J. Klimeš, A. Tkatchenko, D. Alfè, and A. Michaelides, Proc. Natl. Acad. Sci. USA 115, 1724 (2018).

[37] W. L. McMillan, Phys. Rev. 138, A442 (1965).

[38] J. D. Wright, Molecular Crystals, 2nd ed. (Cambridge University Press, Cambridge, 1995).

[39] A. Droghetti, I. Rungger, C. Das Pemmaraju, and S. Sanvito, Phys. Rev. B 93, 195208 (2016).

[40] S. Refaely-Abramson, S. Sharifzadeh, M. Jain, R. Baer, J. B. Neaton, and L. Kronik, Phys. Rev. B 88, 081204(R) (2013).

[41] S. Sharifzadeh, A. Biller, L. Kronik, and J. B. Neaton, Phys. Rev. B 85, 125307 (2012).

[42] J. B. Neaton, M. S. Hybertsen, and S. G. Louie, Phys. Rev. Lett. 97, 216405 (2006)

[43] L. Mitas and R. M. Martin, Phys. Rev. Lett. 72, 2438 (1994).

[44] M. D. Towler, R. Q. Hood, and R. J. Needs, Phys. Rev. B 62, 2330 (2000).

[45] R. J. Needs, M. D. Towler, N. D. Drummond, and P. López Ríos, J. Phys.: Condens. Matter 22, 023201 (2010).

[46] S. J. Clark, M. D. Segall, C. J. Pickard, P. J. Hasnip, M. J. Probert, K. Refson, and M. C. Payne, Z. Kristallogr. 220, 567 (2005).

[47] J. R. Trail and R. J. Needs, J. Chem. Phys. 122, 174109 (2005).

[48] J. R. Trail and R. J. Needs, J. Chem. Phys. 122, 014112 (2005).

[49] S. Azadi, C. Cavazzoni, and S. Sorella, Phys. Rev. B 82, 125112 (2010).

[50] D. Alfè and M. J. Gillan, Phys. Rev. B 70, 161101 (2004).

[51] P. López Rìos, A. Ma, N. D. Drummond, M. D. Towler, and R. J. Needs, Phys. Rev. E 74, 066701 (2006).

[52] F. Calcavecchia and T. D. Kühne, Europhys. Lett. 110, 20011 (2015).

[53] C. J. Umrigar, K. G. Wilson, and J. W. Wilkins, Phys. Rev. Lett. 60, 1719 (1988).

[54] N. D. Drummond and R. J. Needs, Phys. Rev. B 72, 085124 (2005).

[55] T. Kato, Commun. Pure Appl. Math. 10, 151 (1957).

[56] R. T. Pack and W. B. Brown, J. Chem. Phys. 45, 556 (1966).

[57] N. D. Drummond, R. J. Needs, A. Sorouri, and W. M. C. Foulkes, Phys. Rev. B 78, 125106 (2008).

[58] C. Lin, F. H. Zong, and D. M. Ceperley, Phys. Rev. E 64, 016702 (2001).

[59] M. Holzmann, R. C. Clay, III, M. A. Morales, N. M. Tubman, D. M. Ceperley, and C. Pierleoni, Phys. Rev. B 94, 035126 (2016)

[60] S. Chiesa, D. M. Ceperley, R. M. Martin, and M. Holzmann, Phys. Rev. Lett. 97, 076404 (2006).

[61] L. M. Fraser, W. M. C. Foulkes, G. Rajagopal, R. J. Needs, S. D. Kenny, and A. J. Williamson, Phys. Rev. B 53, 1814 (1996). 
[62] A. J. Williamson, G. Rajagopal, R. J. Needs, L. M. Fraser, W. M. C. Foulkes, Y. Wang, and M. Y. Chou, Phys. Rev. B 55, R4851 (1997).

[63] See Supplemental Material at http://link.aps.org/supplemental/ 10.1103/PhysRevB.97.205428 for the value of DMC energies which are obtained at different $r_{S}$.

[64] A. Zen, S. Sorella, M. J. Gillan, A. Michaelides, and D. Alfé, Phys. Rev. B 93, 241118(R) (2016).

[65] M. Nakamura and T. Miyazawa, J. Chem. Phys. 51, 3146 (1969).

[66] B. Civalleri, C. M. Zicovich-Wilson, L. Valenzano, and P. Ugliengo, Cryst. Eng. Commun. 10, 405 (2008).
[67] A. L. Ringer and C. D. Sherrill, Chem. Eur. J. 14, 2542 (2008).

[68] M. R. Kennedy, A. R. McDonald, A. Eugene DePrince, III, M. S. Marshall, R. Podeszwa, and C. David Sherrill, J. Chem. Phys. 140, 121104 (2014).

[69] J. S. Chickos and W. E. Acree, Jr., J. Phys. Chem. Ref. Data 31, 537 (2002).

[70] D. Lu, Y. Li, D. Rocca, and G. Galli, Phys. Rev. Lett. 102, 206411 (2009).

[71] E. Jan Meijer and M. Spirk, J. Chem. Phys. 105, 8684 (1996)

[72] L. A. Curtiss, P. C. Redfern, K. Raghavachari, and J. A. Pople, J. Chem. Phys. 109, 42 (1998). 